Preprint typeset using $\mathrm{LAT}_{\mathrm{E}} \mathrm{X}$ style emulateapj v. 5/2/11

\title{
PHOTOSPHERIC RADIUS EXPANSION IN SUPERBURST PRECURSORS FROM NEUTRON STARS
}

\author{
L. KEEK \\ National Superconducting Cyclotron Laboratory, Department of Physics \& Astronomy, and Joint Institute for Nuclear Astrophysics, \\ Michigan State University, East Lansing, MI 48824, USA
}

\begin{abstract}
Thermonuclear runaway burning of carbon is in rare cases observed from accreting neutron stars as day-long X-ray flares called superbursts. In the few cases where the onset is observed, superbursts exhibit a short precursor burst at the start. In each instance, however, the data was of insufficient quality for spectral analysis of the precursor. Using data from the propane anti-coincidence detector of the PCA instrument on $R X T E$, we perform the first detailed time resolved spectroscopy of precursors. For a superburst from $4 \mathrm{U} 1820-30$ we demonstrate the presence of photospheric radius expansion. We find the precursor to be 1.4-2 times more energetic than other short bursts from this source, indicating that the burning of accreted helium is insufficient to explain the full precursor. Shock heating would be able to account for the lacking energy. We argue that this precursor is a strong indication that the superburst starts as a detonation, and that a shock induces the precursor. Furthermore, we employ our technique to study the superexpansion phase of the same superburst in greater detail.

Subject headings: accretion, accretion disks - methods: numerical — nuclear reactions, nucleosynthesis, abundances — stars: neutron — X-rays: binaries — X-rays: bursts
\end{abstract}

\section{INTRODUCTION}

Superbursts are rare day-long X-ray flares observed from accreting neutron stars in low-mass X-ray binaries (LMXBs, Cornelisse et al. 2000; Strohmaver \& Brown 2002). They are attributed to runaway thermonuclear fusion of carbon in an approximately $100 \mathrm{~m}$ thick layer below the neutron star surface (Cumming \& Bildsten 2001). Superbursts share some properties of the frequently detected short Type I X-ray bursts (durations of $\sim 10-100 \mathrm{~s}$ ), which result from runaway burning of hydrogen and/or helium accreted onto the neutron star from the binary companion (e.g., Lewin et al. 1993). For example, both have a fast rising light curve, followed by a slow decay during which the X-ray spectrum exhibits cooling. Superbursts, however, are 1000 times more energetic, last 1000 times longer, and occur 1000 times less frequently than the short bursts (e.g., Keek \& in 't Zand 2008).

Currently 22 (candidate) superbursts are known from 13 sources (see, e.g., Keek et al. 2012 for an overview). All detections are performed with observatories in low earth orbit, which implies observations are frequently interrupted by Earth occultations and passages through the South-Atlantic Anomaly. Often the start of a superburst is not observed. In some cases this makes it difficult to prove the superburst nature of an event: these are referred to as candidates (e.g., in 't Zand et al. 2004). Only in 6 cases is there an unambiguous detection of the onset. In all these cases a precursor burst is present at the superburst start (Strohmaver \& Brown 2002; Strohmaver \& Markwardt 2002; in 't Zand et al. 2003, 2004). In previous studies the term "precursor" has also been used to describe a burst that precedes the superburst by two minutes (Kuulkers et al. 2002b), or when two of these events are visible in the superburst rise (Strohmaver \& Brown 2002). Because these likely have a different physical origin, we restrict our study

keek@nscl.msu.edu to those precursor bursts that immediately precede and transition into the superburst.

The most detailed precursor observations have been performed with the Proportional Counter Array (PCA) on the Rossi X-ray Timing Explorer (RXTE), and are from superbursts of 4U 1820-30 (Strohmaver \& Brown 2002) and 4U 1636-53 (Strohmayer \& Markwardt 2002).

$4 \mathrm{U} 1636-53$ is a prolific burster that has exhibited many different modes of thermonuclear burning of hydrogen and helium (e.g., Hoffman et al. 1977; van Paradijs et al. 1986; Revnivtsev et al.|2001), as well as carbon burning in 4 superbursts (Wiinands 2001; Strohmaver \& Markwardt 2002; Kuulkers et al. 2004; Kuulkers 2009).

$4 \mathrm{U} 1820-30$ is an ultracompact X-ray binary (UCXB, Stella et al. 1987), implying that the material accreted from the donor star is likely helium rich (Rappaport et al. 1987, see also Cumming 2003). Its persistent flux is observed to vary on a timescale of approximately 171 days (Priedhorsky \& Terrell 1984; Chou \& Grindlay 2001), and X-ray bursts are only detected during periods of low flux (e.g., Cornelisse et al. 2003). Two (candidate) superbursts have been observed (Strohmayer \& Brown 2002; in 't Zand et al. 2011b). The first superburst from this source was observed with the PCA, and used to study the changing properties of the accretion disk during the superburst (Ballantyne \& Strohmaver 2004), the occurrence of superexpansion of the photosphere (in't Zand \& Weinberg 2010), and achromatic variability in the light curve (in 't Zand et al. 2011a).

The precursors resemble short X-ray bursts, and the light curve exhibits two peaks, similar to photospheric radius expansion (PRE) bursts (Tawara et al. 1984; Lewin et al. 1984). During powerful X-ray bursts, when the luminosity reaches the Eddington limit, the photon pressure is sufficient to (temporarily) push out the photosphere, causing the surface temperature to drop. The lower temperature results in a smaller part of the black- 
body flux to fall within the energy band in which many $\mathrm{X}$-ray detectors are sensitive, producing a dip in the light curve. Unfortunately no spectral data of sufficient quality have been available to confirm the PRE nature of superburst precursors.

An alternative explanation for the double peaked nature of precursors has been put forth. Carbon burning in a superburst that proceeds as a detonation drives a shock to the surface (Weinberg et al. 2006), where it produces a shock-breakout peak in the light curve, followed by a burst from the shock-ignited burning of hydrogen and helium in the atmosphere. Weinberg \& Bildsten (2007) argue that the two peaks are separated by a thermal timescale of seconds, similar to the two peaks observed in precursors. Keek \& Heger (2011), however, show that the fallback of the shock-accelerated atmosphere alone dissipates enough energy to power a bright precursor on a dynamical timescale of $10 \mu \mathrm{s}$. At the same time any hydrogen or helium present in the atmosphere ignites and contributes to the precursor energetics (Keek et al. 2012). In this scenario the shock breakout and the subsequent burst are too close in time to be distinguished as two peaks, but the burst is bright enough to cause radius expansion.

To test the different models for superburst precursors, we devise a technique using anti-coincidence data of the PCA to perform time resolved spectroscopy on the two superbursts observed with this instrument from, respectively, $4 \mathrm{U} 1820-30$ and $4 \mathrm{U} 1636-53$. This is the first time precursors are studied in detail, and we discuss the implications for the nature of precursors and of superburst ignition.

\section{OBSERVATIONS}

RXTE (Levine et al. 1996) was launched in December 1995 and observed the X-ray sky until January 2012. It carries the PCA (Jahoda et al. 2006), which has a band pass of 2 to $60 \mathrm{keV}$, and a total collecting area of $6500 \mathrm{~cm}^{2}$. As part of its science program the PCA frequently observed LMXBs, and it detected thousands of short X-ray bursts (e.g., Galloway et al. 2008) as well as the two mentioned superbursts.

The PCA consists of 5 identical collimated Proportional Counter Units (PCUs). Each PCU contains three xenon-filled layers, which act as the main counter, and are sensitive in the $2-60 \mathrm{keV}$ energy range. On top of the xenon detector is a propane-filled anti-coincidence detector. It provides additional sensitivity between 1.8 $3.5 \mathrm{keV}$ with peak sensitivity at $2.5 \mathrm{keVI}$, but little calibration information is available. Data from the xenon layers are typically available per layer and PCU at high time resolution and with spectral information, whereas for the propane layer only a count rate for all PCUs combined is available at $0.125 \mathrm{~s}$ time resolution without spectral information.

At the time of both superburst observations there were problems that resulted in a loss of data. At the time of the $4 \mathrm{U}$ 1820-30 superburst observation, $R X T E$ was troubled by a malfunction that prevented part of the data from being transmitted to the ground (Strohmayer \& Brown 2002). During the 4U 1636-53 su- perburst the data buffer overflowed, causing a loss of data (Strohmaver \& Markwardt 2002). Most notably, high time resolution spectral data were lost in both cases. During the superburst rises only Standard1 and Standard2 data are available, which have a time resolution of $0.125 \mathrm{~s}$ and $16 \mathrm{~s}$, respectively. Only the latter mode contains spectral information, but its time resolution exceeds the duration of the precursors. We are, therefore, limited to using Standard1 data. Additionally, the propane count rate is available at $0.125 \mathrm{~s}$ time resolution.

Because of the different energy responses of the xenon and propane layers, count rates from the two can be regarded as representing two energy channels. This allows for fitting a spectral model with two parameters, such as the black-body model that is often used to describe burst spectra (Swank et al. 1977). The propane signal, however, is not calibrated for this purpose (Jahoda et al. 2006). To investigate the energy response in these two "channels" to a black-body spectrum, we perform time resolved spectroscopy on several short bursts from the two superbursters for which event data with a minimum time resolution of $125 \mu$ s and with 64 energy channels are available.

We select short bursts from version 0.51 of the MultiINstrument Burst Archive 2 (MINBAR, Keek et al. 2010), which contains the results of the analysis of 4,192 bursts observed from 72 sources with the PCA (Galloway et al. 2008) and the Wide-Field Camera's on the BeppoSAX observatory (Cornelisse et al. 2003). For the purpose of this study we only use PCA bursts.

The gain settings of the PCUs were changed several times during the mission. To be able to compare count rates, we restrict ourselves to bursts that were observed with the same detector gain as the respective superbursts. Furthermore, the gain was slightly different for each PCU (up to 1\%, Jahoda et al. 2006). The propane data, however, are stored as an aggregate, and not per PCU. We, therefore, use the propane and xenon data of all active PCUs.

The number and configuration of active PCUs changes per observation, and due to the impact of micrometeorites PCU 0 and 1 lost their propane layer on, respectively, May 132000 and December 252006 (Jahoda et al. 2006). We, therefore, report xenon and propane count rates per active detector. An active detector that has lost its propane layer is still counted as an active detector for determining the xenon count rate, but not for the propane rate.

There are small changes in the gain and instrument response over time. For example, xenon is known to have leaked into the propane layer. When analyzing the short bursts, we generate response matrices for each burst to take into account the instrument response and the configuration of active PCUs at a particular time.

For spectra and count rates we subtract the background measured during a time interval before each (super)burst. This yields the net burst rates and spectra, and removes the astrophysical background as well as the instrumental background. Here we assume both backgrounds remain constant during the burst. We further discuss this in Section 4.1, but especially in the burst

${ }^{1}$ We refer to http://heasarc.gsfc.nasa.gov/docs/xte/appendix_f.html for XTE Technical Appendix F.

2 See http://users.monash.edu.au/ dgallow/minbar for more details. 
TABLE 1

13 PRE Bursts from 4U 1820-30 and a Superburst Precursor (Bottom)

\begin{tabular}{lccc}
\hline Obs.ID & $\begin{array}{c}\text { Time } \\
\text { (MJD) }\end{array}$ & $\begin{array}{c}\text { Fluence } \\
\left(10^{-7} \mathrm{erg}\right. \\
\left.\mathrm{cm}^{-2}\right)\end{array}$ & $\begin{array}{c}\text { Xenon counts } \\
\left(10^{4} \mathrm{c} \mathrm{cm}^{-2}\right. \\
\left.\mathrm{PCU}^{-1}\right)\end{array}$ \\
\hline $40017-01-24-00$ & 52794.73813 & $3.6 \pm 0.1$ & $3.20 \pm 0.08$ \\
$70030-03-04-01$ & 52802.07557 & $4.0 \pm 0.1$ & $3.37 \pm 0.07$ \\
$70030-03-05-01$ & 52805.89566 & $4.1 \pm 0.2$ & $3.31 \pm 0.08$ \\
$90027-01-03-05$ & 53277.43856 & $3.7 \pm 0.1$ & $3.24 \pm 0.08$ \\
$94090-01-01-02$ & 54948.82124 & $3.2 \pm 0.2$ & $3.01 \pm 0.14$ \\
$94090-01-01-05$ & 54950.70281 & $3.5 \pm 0.1$ & $3.22 \pm 0.10$ \\
$94090-01-02-03$ & 54956.77470 & $3.3 \pm 0.1$ & $3.08 \pm 0.11$ \\
$94090-01-02-02$ & 54958.73998 & $3.2 \pm 0.2$ & $2.98 \pm 0.14$ \\
$94090-01-04-00$ & 54978.32149 & $3.7 \pm 0.2$ & $3.24 \pm 0.14$ \\
$94090-01-04-01$ & 54978.49489 & $3.7 \pm 0.2$ & $3.3 \pm 0.2$ \\
$94090-01-05-00$ & 54981.18728 & $3.8 \pm 0.1$ & $3.21 \pm 0.09$ \\
$94090-02-01-00$ & 54994.53418 & $3.2 \pm 0.2$ & $2.95 \pm 0.13$ \\
$94090-02-01-00$ & 54994.61301 & $3.6 \pm 0.1$ & $3.15 \pm 0.10$ \\
\hline $30057-01-04-08$ & 51430.07423 & $6-8$ & $4.59 \pm 0.06$ \\
\hline
\end{tabular}

peak the signal is expected to be dominated by the burst, so small changes in the background should not be detrimental to our analysis.

All extracted data products, including spectra and light curves, are corrected for dead time using the prescription provided by the instrument team. Data required for this correction are available at a resolution of $0.125 \mathrm{~s}$. When calculating the correction we take into account that some of the active PCUs may have lost their propane layer.

\section{RESULTS}

\subsection{U 1820-30}

On $9 / 9 / 1999$ the PCA detected a superburst from 4U 1820-30 (Strohmaver \& Brown 2002). The superburst was observed for three hours, and the flux continued to decrease when the observation ended. Two events with PRE-like peaks and dips are visible in the light curve. We consider the first to be the precursor. MINBAR lists 14 bursts from this source detected with the PCA, 13 of which were observed with the same high voltage settings as the superburst (Table 11). All bursts exhibit PRE. We perform time-resolved spectroscopy on the bursts to calibrate the xenon and propane count rates such that these can be used to analyze the superburst precursor.

\subsubsection{Analysis of short bursts}

Net burst spectra are extracted at a resolution of $0.125 \mathrm{~s}$ in the rise and at the peak of the burst. After the peak we decrease the time resolution by a factor 2 every time the count rate per PCU decreases by $\sqrt{2}$, which ensures similar statistics for all spectra. Each spectrum is fit with a black body in the $2.5-25 \mathrm{keV}$ energy range. Absorption by interstellar hydrogen is taken into account using the cross sections of Morrison \& McCammon (1983), while fixing the hydrogen column to $N_{\mathrm{H}}=0.25 \times 10^{22}$ (Asai et al. 2000). The fit yields the black-body temperature, $k T$, and normalization, $K_{\mathrm{bb}} \equiv\left(\frac{R}{\mathrm{~km}}\right)^{2}\left(\frac{d}{10 \mathrm{kpc}}\right)^{-2}$, with $R$ the black-body radius and $d$ the distance to the source.

For each time bin we extract the count rate in the propane layer and in the xenon layer (per PCU). We determine the sum of the xenon and propane count rate, $I$,

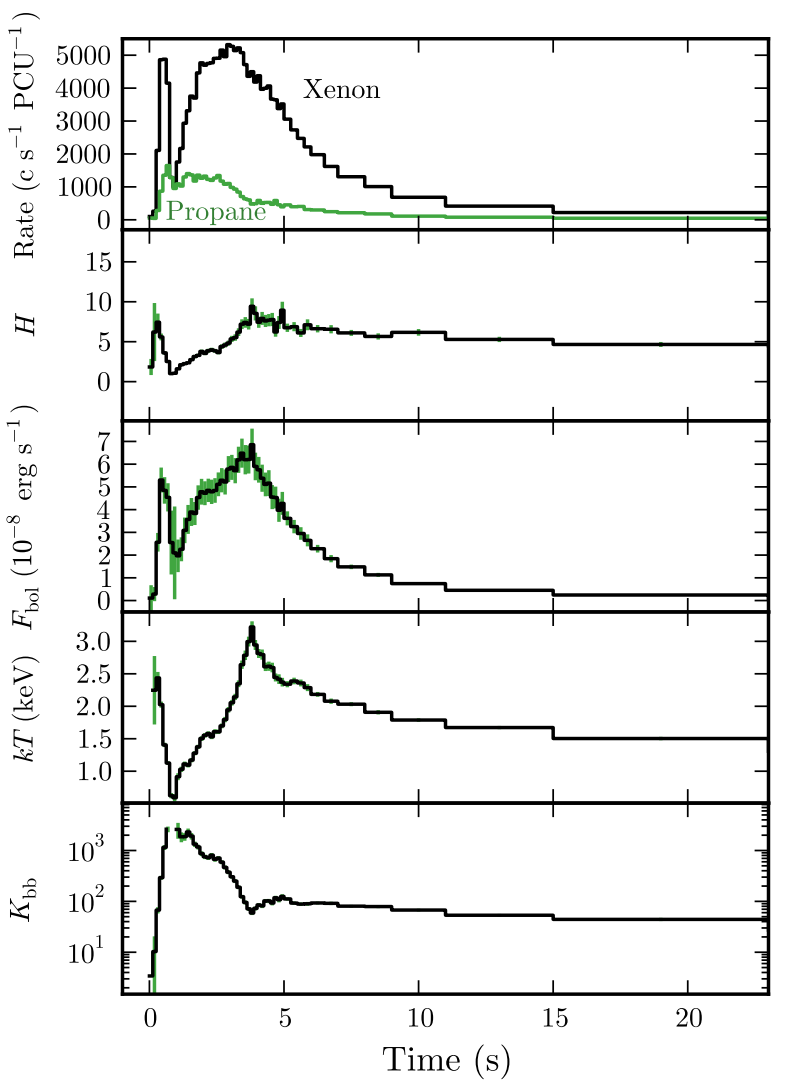

FIG. 1. - For the X-ray burst on MJD 52794 from 4U 1820-30 the count rate in the xenon and the propane layers, hardness $H$ (ratio of xenon and propane counts), and the results of time resolved spectroscopy: the unabsorbed bolometric flux $F_{\text {bol }}$, the black-body temperature $k T$, and the black-body normalization $K_{\mathrm{bb}}$. For the latter we omit two data points with anomalously large values and errors. Vertical (green) lines indicate the $1 \sigma$ uncertainties per time bin (no uncertainties are indicated for the rates).

and the hardness ratio of the two rates, $H$. An example of the evolution during a burst of these quantities and the spectral fit parameters is shown in Figure 1.

$4 \mathrm{U}$ 1820-30 experiences rather strong radius expansion during its bursts, which pushes a large part of the emission out of the PCA band. The peak of the count distribution for a black body lies at an energy of $E_{\max } \simeq 1.59 \mathrm{kT}$. Because of absorption at low energies, the peak of the spectrum is shifted to slightly higher energies. When the black body temperature drops below $k T \simeq 1.6 \mathrm{keV}$ the peak is outside the considered bandpass. The fits become worse around $k T \simeq 1.0 \mathrm{keV}$, resulting in dips and peaks in the bolometric flux. Therefore, our fits are not reliable for $k T \lesssim 1.0 \mathrm{keV}$. In further analysis, we use $k T>1.0 \mathrm{keV}$ when deriving relations between the different parameters. Furthermore, in the tail of the bursts the net burst counts are strongly reduced, and the relative uncertainty in $H$ and $I$ become large. In our analysis we omit data points where the relative uncertainty in $H$ exceeds $50 \%$.

The hardness ratio traces changes in the spectral shape, which for a black body is set by $k T$. We determine the relation between $k T$ and $H$ by fitting a straight line (Figure 2). To determine the goodness of fit we use the error in $H$, because it is typically larger than the error in $k T$. To ensure the latter, we exclude data points where 


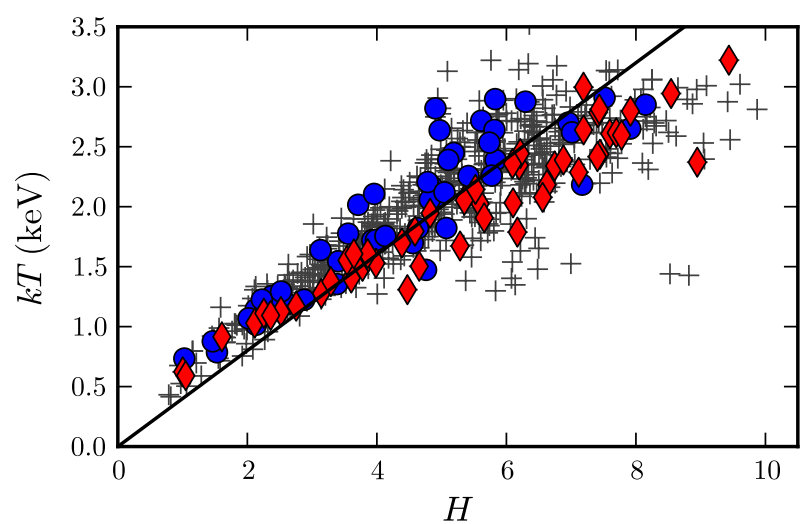

FIG. 2.- Hardness (ratio of xenon and propane counts) versus the black-body temperature $k T$ from time-resolved spectroscopy of 13 PRE bursts. Crosses indicate the data points for all bursts (no uncertainties implied), whereas disks and diamonds are for two bursts on MJD 52794.7 and MJD 54994.5, respectively. The solid line is the best linear fit to the data with $k T>1.0 \mathrm{keV}$.

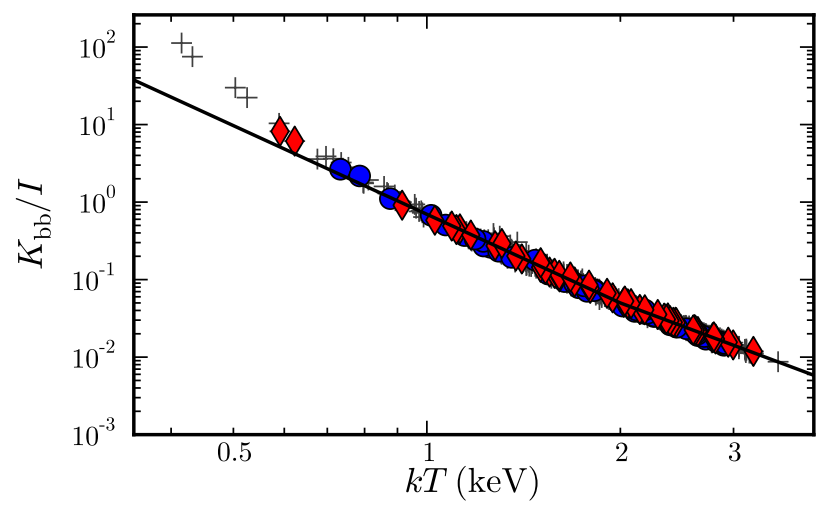

FIG. 3.- Black-body temperature $k T$ versus the ratio of the black-body normalization $K_{\mathrm{bb}}$ and the total count rate $I$ for 13 PRE bursts. See Figure 2 for an explanation of the symbols. The solid line is the best fit broken power-law to the data with $k T>$ $1.0 \mathrm{keV}$.

the relative uncertainty in $k T$ exceeds $10 \%$. The best fit line is

$$
k T=(0.40 \pm 0.06) H \mathrm{keV} .
$$

To obtain $\chi_{\nu}^{2}=1$ a systematic error of $16 \%$ needs to be added in quadrature to the uncertainty of the data points. This describes the spread in the distribution of both individual bursts and all bursts combined. Some individual bursts have a smaller spread (Figure 2), but the spread between such bursts is substantial, so this does not aid us in improving the fit. Furthermore, the fitted line is defined to go through zero. Including an offset, the best fit conforms better to the points with $k T \lesssim 1.0 \mathrm{keV}$ (which are excluded from the fit), but the fit statistics are not improved; the best fit value is within $1 \sigma$ consistent with 0.0 . Furthermore, competition between the fit parameters leads to large uncertainties in the parameters.

Given $k T$, which sets the spectral shape, $K_{\mathrm{bb}}$ is linearly proportional to $I$. We determine the ratio $K_{\mathrm{bb}} / I$, and we find that as a function of $k T$ it follows a relation that is close to a power law (Fig. 3). We fit a broken power law with the break at $2 \mathrm{keV}$, which is approximately the

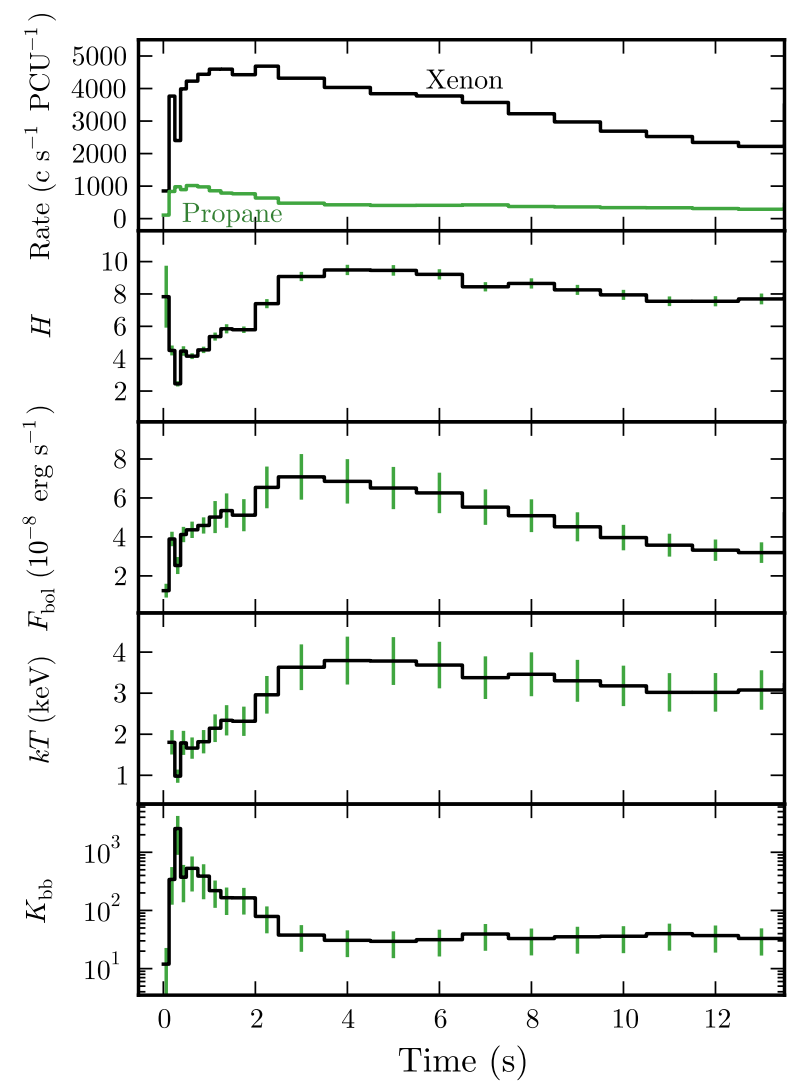

Fig. 4.- Similar to Figure 1 for the precursor of the $4 \mathrm{U}$ 1820-30 superburst.

center of the region over which we fit, and we define the power-law normalization at the location of the break. The best fit is

$$
\frac{K_{\mathrm{bb}}}{I}=(5.0 \pm 0.4) \times 10^{-2}\left(\frac{k T}{2 \mathrm{keV}}\right)^{-\Gamma},
$$

with $\Gamma=3.8 \pm 0.2$ for $k T<2 \mathrm{keV}$ and $\Gamma=3.10 \pm$ 0.07 for $k T \geq 2 \mathrm{keV}$. The power-law index is $1.4 \sigma$ from 3.0 for $k T>2 \mathrm{keV}$ (where the peak of the black-body counts spectrum is within the xenon detector band-pass), which is the expected value for a Planck counts spectrum. Performing a fit of a single power law with the index fixed to this value leads, however, to a larger error in the prefactor.

For all bursts we determine the total number of xenon counts per PCU and the unabsorbed bolometric fluence (Table [1).

\subsubsection{Analysis of the superburst precursor}

We divide the first $13.5 \mathrm{~s}$ of the superburst into time intervals, using $0.125 \mathrm{~s}$ bins at the start to resolve the rise and dip in the count rate at the highest available resolution. We double the duration of the time intervals several times to get similar uncertainties in the data points of the entire precursor. The tail is resolved at $1 \mathrm{~s}$ resolution. We extract $H$ and $I$, and use equations (11, 2) to derive the black body parameters (Figure 4). At the start of the precursor we can clearly see $K_{\mathrm{bb}}$ increasing, accompanied by the dip in $k T$. This is indicative of PRE.

The unabsorbed bolometric black-body flux $F_{\text {bol }}=$ 


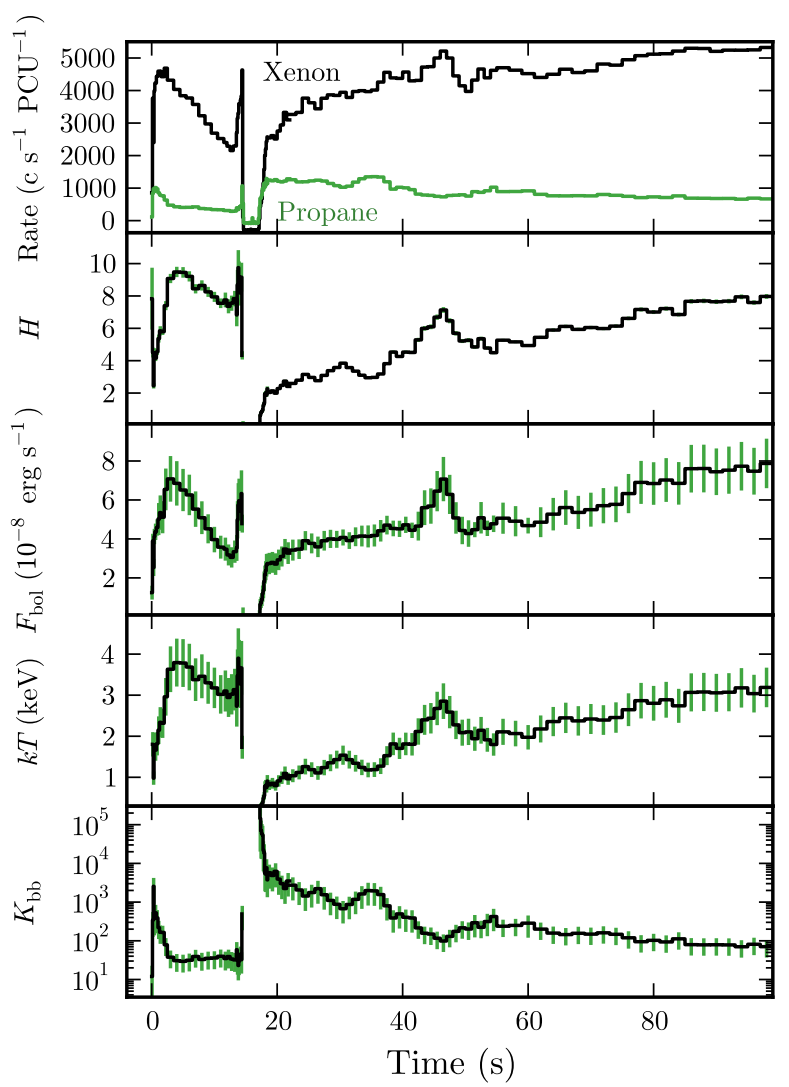

FIG. 5.- Similar to Figure 1 for the start of the $4 \mathrm{U} 1820$ 30 superburst, including the precursor. We omit the data points where the xenon rate drops below 0 .

$\frac{R^{2}}{d^{2}} \sigma T^{4}$ can be expressed in terms of $I$ and $k T$ using equations (1], 2):

$$
F_{\text {bol }}=I\left(\frac{k T}{2 \mathrm{keV}}\right)^{\Gamma}(8.6 \pm 0.7) \times 10^{-12} \mathrm{erg} \mathrm{s}^{-1} \mathrm{~cm}^{-2}
$$

with $\Gamma=0.2 \pm 0.2$ for $k T<2 \mathrm{keV}$ and $\Gamma=0.90 \pm$ 0.07 for $k T \geq 2 \mathrm{keV}$. There is a brief dip in $F_{\text {bol }}$ at the moment of maximum radius expansion (Figure 4). This is most likely caused by the peak of the Planck spectrum being outside the observable band when the temperature drops to $\sim 1 \mathrm{keV}$, and not due to a genuine drop in the bolometric flux.

The light curve as shown in Figure 4 , i.e. the first $13.5 \mathrm{~s}$ of the precursor, has an unabsorbed bolometric fluence of $(6.8 \pm 1.1) \times 10^{-7} \mathrm{erg} \mathrm{cm}^{-2}$. After $13.5 \mathrm{~s}$, the light curve rises to the superburst peak. If we were to consider the precursor as an isolated burst, without the interruption by the superburst, the flux would continue to decay. We fit $F_{\text {bol }}$ with an exponential, starting at $t=$ $5.5 \mathrm{~s}$, when the flux drops below $90 \%$ of the peak flux (cf. Galloway et al. 2008). We find an exponential decay time scale $\tau=10 \pm 3 \mathrm{~s}$. Using this time scale to extrapolate beyond $13.5 \mathrm{~s}$, we estimate an additional contribution to the precursor fluence of $(1.5 \pm 1.0) \times 10^{-7} \mathrm{erg} \mathrm{cm}^{-2}$, giving a total of $(8 \pm 2) \times 10^{-7} \mathrm{erg} \mathrm{cm}^{-2}$.

\subsubsection{Analysis of the second PRE phase}

The superburst was only spectrally analyzed from $100 \mathrm{~s}$ after the (precursor) onset by Strohmaver \& Brown
(2002). in't Zand \& Weinberg (2010) study the first $100 \mathrm{~s}$ at a time resolution of $16 \mathrm{~s}$. We use our technique to analyze this time interval at higher time resolution (Figure [5). Variability is present in $F_{\text {bol }}$, most notably around $t \simeq 47 \mathrm{~s}$, which exhibits an anticorrelation between $k T$ and $K_{\mathrm{bb}}$. The variability is present in the signal from all active PCUs.

The superburst shows very strong radius expansion: an order of magnitude larger than the radius expansion during the precursor. Assuming a distance of $7.6 \pm 0.4 \mathrm{kpc}$ (Kuulkers et al. 2003), we estimate the velocity of the photosphere during expansion by dividing the change in radius between consecutive data points by the change in time, and find as largest values $(2.0 \pm 0.7) \times 10^{2} \mathrm{~km} \mathrm{~s}^{-1}$ during the precursor (at $t=0.3 \mathrm{~s}$ in Figure 4) and $(1.6 \pm 0.6) \times 10^{4} \mathrm{~km} \mathrm{~s}^{-1}$ during the superexpansion phase (at $t=14 \mathrm{~s}$ in Figure 5). Note that both velocities are each only found from the difference between two data points. Velocity measurements directly preceding are substantially lower, indicating strong acceleration. Potentially the velocity of the photosphere increased further during the superexpansion, when the signal was lost.

\subsubsection{Comparison of fluence of the precursor and short bursts}

Strohmayer \& Brown (2002) performed a timeresolved spectral analysis of the superburst from $4 \mathrm{U}$ 1820-30 using Standard2 data at $16 \mathrm{~s}$ resolution. They start at $100 \mathrm{~s}$, because at earlier times the spectral changes are not well resolved by $16 \mathrm{~s}$ time bins. At $t=100 \mathrm{~s}$ they report $k T \simeq 2.6 \mathrm{keV}$ and $K_{\mathrm{bb}} \simeq 115$. We find at that time $k T=3.2 \pm 0.5 \mathrm{keV}$ and $K_{\mathrm{bb}}=(7 \pm 4) \times 10^{1}$, which differ by $1.1 \sigma$ and $1.2 \sigma$, respectively, from the measurement by Strohmaver \& Brown 2002 (see also in't Zand \& Weinberg 2010). The hardness ratio is $H=7.88 \pm 0.14$, which is at the upper end of the interval for which the linear correlation between $k T$ and $H$ describes the data well, whereas at higher values of $H$ the derived $k T$ values lie somewhat below that line (Figure 2).

If we take this as a sign that our derived $k T$ values are too large by a factor $3.2 / 2.6$, we can scale equation (11) by the inverse of this factor. Redoing the analysis with reduced temperatures yields a fluence of $(5.7 \pm 0.8) \times 10^{-7} \mathrm{erg} \mathrm{cm}^{-2}$, and $(7.8 \pm 1.5) \times 10^{-7} \mathrm{erg} \mathrm{cm}^{-2}$ including an extrapolated tail. The effect on $K_{\text {bb }}$ can be estimated from equation (2) to be approximately a factor 2 , implying the radius is larger by a factor 1.4 .

The maximum burst fluence we measure is $(4.1 \pm 0.2) \times$ $10^{-7} \mathrm{erg} \mathrm{cm}^{-2}$ (Table 11). This is $2.5 \sigma$ away from our original fluence measurement and $2.0 \sigma$ from the one with the scaled $k T$; here we did not include the estimate of the extrapolated tail fluence, as this increases the uncertainty. Whereas the inherent uncertainty in the method we employ does not allow for a strongly significant difference in the precursor fluence and the maximum burst fluence, a direct comparison of the integrated burst counts clearly shows the precursor to be more energetic (Figures 1, 44). The normal bursts have at most $(3.37 \pm 0.07) \times 10^{4} \mathrm{c} \mathrm{cm}^{-2}$ (Table1), whereas the precursor (without extrapolated tail) has $(4.59 \pm 0.06) \times 10^{4} \mathrm{c} \mathrm{cm}^{-2}$, which is a $17 \sigma$ difference. The precursor has at least 


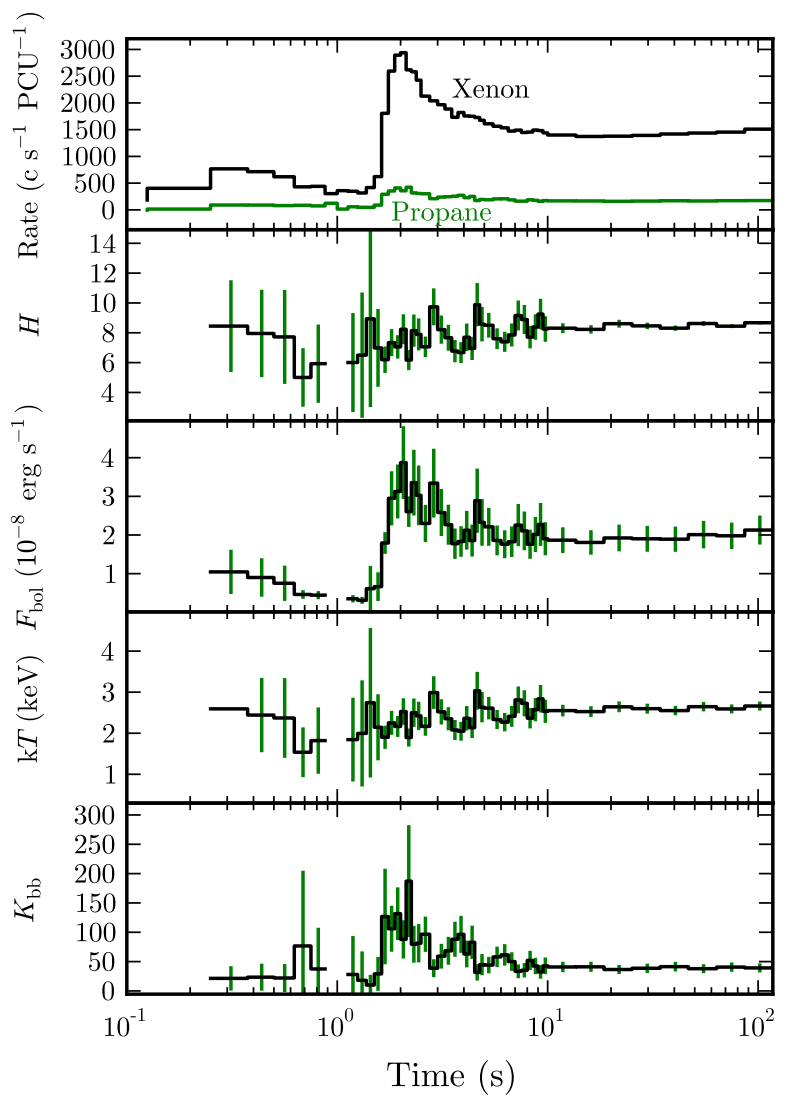

FIG. 6. - Similar to Figure 1 for the precursor of the $4 \mathrm{U} 1636$ 53 superburst. Data points with particularly large uncertainties in the hardness are omitted.

$1.36 \pm 0.03$ times more counts, and between $1.4 \pm 0.2$ (scaled, no tail) and $2.0 \pm 0.5$ (original, with tail) more fluence. From the difference in count rates, we conclude that the precursor is significantly and substantially more energetic than the normal bursts.

\section{2. $4 U 1636-536$}

On 2/22/2001 the PCA observed a superburst from $4 \mathrm{U}$ 1636-53. The light curve exhibits a double peak at the onset (Figure 6). The number of active PCUs with a propane layer was the same as during the superburst from $4 \mathrm{U}$ 1820-30 (3), but the observed propane count rate was less than half. The uncertainty in the hardness ratio, $H$, is large in the first few seconds. Coincident with the dip in the rate, a hint of a dip in $H$ may be present around $1 \mathrm{~s}$ after the onset, indicating a dip in the black-body temperature, which would be consistent with PRE. The uncertainties, however, are far too large to draw any conclusions. We applied the same analysis as for $4 \mathrm{U}$ 1820-30, using three PRE bursts detected in the preceding month with the PCA (Table 2). We find relations analogous to Equations (11, 2):

$$
k T=(0.307 \pm 0.012) H \mathrm{keV},
$$

which requires a systematic uncertainty of $7 \%$ to obtain $\chi_{\nu}^{2}=1$, and

$$
\frac{K_{\mathrm{bb}}}{I}=(4.9 \pm 0.7) \times 10^{-2}\left(\frac{k T}{2 \mathrm{keV}}\right)^{-\Gamma},
$$

TABLE 2

3 PRE Bursts from 4U 1636-53 and a Superburst Precursor (Bottom)

\begin{tabular}{lccc}
\hline Obs.ID & $\begin{array}{c}\text { Time } \\
\text { (MJD) }\end{array}$ & $\begin{array}{c}\text { Fluence } \\
\left(\begin{array}{c}10^{-7} \text { erg } \\
\left.\mathrm{cm}^{-2}\right)\end{array}\right.\end{array}$ & $\begin{array}{c}\text { Xenon counts } \\
\left(10^{4} \mathrm{c} \mathrm{cm}^{-2}\right. \\
\left.\mathrm{PCU}^{-1}\right)\end{array}$ \\
\hline $50030-02-04-00$ & 51937.11615 & $4.93 \pm 0.12$ & $4.25 \pm 0.09$ \\
$50030-02-05-00$ & 51942.10027 & $4.78 \pm 0.14$ & $4.49 \pm 0.10$ \\
$50030-02-05-01$ & 51941.87561 & $4.39 \pm 0.10$ & $3.87 \pm 0.08$ \\
\hline $50030-02-08-01$ & 51962.70299 & $3.5 \pm 0.9$ & $2.69 \pm 0.06$ \\
\hline
\end{tabular}

with $\Gamma=4.2 \pm 0.3$ for $k T<2 \mathrm{keV}$ and $\Gamma=2.6 \pm 0.3$ for $k T \geq 2 \mathrm{keV}$. These values are consistent with those obtained for $4 \mathrm{U} 1820-30$. Using these relations we determine the black-body parameters for the superburst precursor. There is a hint of weak radius expansion, but the errors are too large to constrain whether PRE is present (Figure 6).

Because the rise of the superburst emission in the light curve is slow compared to the superburst from $4 \mathrm{U} 1820$ 30 , it is more difficult to separate the precursor flux from the superburst flux. We determine the total counts and the fluence up to the local minimum in the flux at $t=$ $18 \mathrm{~s}$ (Table 2). For the three short bursts, the counts and fluence at $t>18 \mathrm{~s}$ contribute at most $15 \%$ of the total. Furthermore, part of the counts and fluence at $t<18 \mathrm{~s}$ may be attributed to the superburst. Therefore, we regard the counts and fluence up to $t=18 \mathrm{~s}$, increased by $15 \%$, to be an upper limit to the precursor emission. Because of the large uncertainty in the flux, the fluence is not significantly different from the fluence of the short bursts, but the counts are significantly lower.

\section{DISCUSSION}

We devised a method to perform time-resolved spectral analysis of superburst precursors using data from the anti-coincidence detector of the PCA on $R X T E$. For a superburst of $4 \mathrm{U} \quad 1820-30$ we clearly see PRE, but for $4 \mathrm{U} 1636-53$ the data quality is insufficient to argue whether radius expansion occurred. In this section we first discuss the possibility for improvements to our spectral analysis technique. Then we discuss the implications of our results.

\subsection{Improvements to the analysis method}

The spectrum of Type I bursts is typically well fit by a black body (Swank et al. 1977). Using the propane and xenon counts we have precisely enough energy "channels" to fit this model. There are improvements over a pure black body that we cannot implement. Corrections are proposed in the form of a so-called color correction factor for the black-body temperature of typically several tens of percents (e.g., Suleimanov et al. 2011). Furthermore, we obtain the net burst spectrum by subtracting the persistent background from a pre-burst time interval. Here we assume that the pre-burst emission is dominated by the accretion disk (e.g., Kuulkers et al. 2002a), and that this emission remains unchanged during the burst. When there is strong radius expansion this may no longer be correct. Indeed, the count rate drops below the pre-burst level during the long PRE phase in the superburst rise of 4U 1820-30 (Figure 5). Strohmaver \& Brown (2002) find that in the tail the qualitative results do not differ greatly between assuming a constant persistent flux and including the persistent spectrum in the fit. 
Strohmayer \& Brown (2002) include in their spectral model for the superburst of $4 \mathrm{U}$ 1820-30 an emission line and an absorption edge. These features possibly result from reflection of burst emission off the accretion disk. Later studies have successfully used spectral models including a disk reflection component (Ballantyne \& Strohmaver 2004; in't Zand \& Weinberg 2010). We do not include these in the model when fitting the normal bursts. Even though they reach the Eddington limit, the radius expansion is smaller for the normal bursts than during the superburst. Furthermore, during the superburst longer time intervals can be chosen for the spectral analysis, which allows these spectral features to become significant. This is not the case during the short bursts.

There is considerable spread in the relation between the hardness ratio and the temperature (Figure 2). The width of the distribution is due to the spread in the data points of individual bursts and the spread between bursts. We investigate the possibility of improving on the fitted linear relation. The xenon detector's effective area is well known, but the area is poorly constrained for the propane layer. An effective area curve, that has the shape of a power law with a smooth low-energy cut-off, is presented in the XTE Technical Appendix F. We employ this curve and a black-body model in an attempt to reproduce the propane counts for the short bursts. Whereas at low count rates there is reasonable agreement, especially at the higher count rates during the burst peaks there are strong deviations from the observed count rates, with a large spread in the deviations. The effective area curve is, therefore, unusable to improve the $H-k T$ relation.

\subsection{Precursor from $4 U$ 1820-30}

The start of the PCA superburst from 4U 1820-30 exhibits two instances of a peak and dip in the light curve (Strohmaver \& Brown 2002). The first we refer to as the precursor, whereas the second has been identified as a period of superexpansion (in’t Zand \& Weinberg 2010, see Section 4.3).

The precursor clearly exhibits PRE (Figure 4). The peak of the black-body temperature is reached $4 \mathrm{~s}$ after the onset (the "touch-down point"). The peak values of the black-body parameters are similar to those of the short bursts (Figure 11). The precursor has, however, a longer decay time and a larger fluence. It is more energetic by a factor $1.4-2$ than the most energetic of the short bursts we analyzed (Table 11).

If a sufficiently large column of carbon ignites, burning is predicted to proceed as a detonation (Weinberg et al. 2006). A shock is generated, and travels to the surface (Weinberg \& Bildsten 2007). This produces a brief shock breakout peak in the light curve. The shock heats the atmosphere, which produces a precursor burst. Furthermore, it triggers the burning of hydrogen and helium, which adds to the energetics of the precursor (Weinberg \& Bildsten 2007). Keek \& Heger (2011) showed that most of the heat from the shock is released upon the fallback of shock-accelerated layers. At that moment any available hydrogen/helium ignites (Keek et al. 2012). The heat released by the shock is expected to be of similar magnitude as that produced by helium burning in the atmosphere.
The superburst took place at a level of persistent flux where short bursts are observed from this source (Zdziarski et al. 2007). The amount of helium present in the neutron star atmosphere at the moment of the superburst depends on the time since the last occurrence of a short burst. Unfortunately this burst was not observed, but the fuel column is at most of similar size as the most energetic short burst.

Given that we observe the precursor to have a larger fluence than the short bursts, the burning of helium is insufficient to power the precursor. We propose shock heating as the most likely source to complete the precursor energetics. This provides a strong indication that the superburst indeed proceeded as a detonation, and confirms the scenario of a shock-induced precursor.

The two peaks in the $2-60 \mathrm{keV}$ precursor light curve are caused by PRE; they are not two peaks intrinsic to the (bolometric) signal. This disproves the suggestion that the first peak is caused by shock breakout and the second is powered by a helium flash (Weinberg \& Bildsten 2007). A scenario where both these events are separated in time by an interval that is shorter than the $0.125 \mathrm{~s}$ resolution of the data, such as the dynamical timescale of $\sim 10^{-5} \mathrm{~s}$, is consistent with the observations (Keek \& Heger 2011).

\subsection{Superexpansion phase}

The 4U 1820-30 superburst is one of only few bursts to exhibit superexpansion, where briefly the blackbody radius increases by as much as a factor 100 (in't Zand \& Weinberg 2010). It is speculated to be caused by the ejection of a shell of material from the neutron star surface. The superexpansion disappears from the observations when the shell turns optically thin, and a lower lying layer becomes visible (in't Zand \& Weinberg 2010). This lower layer is moderately expanded and is observed to settle over time. This superburst has the longest period of moderate expansion observed to date of close to $1400 \mathrm{~s}$.

Using our method we can study the superexpansion of the superburst at much higher time resolution (Figure 5). Using a distance of $7.6 \mathrm{kpc}$ (Kuulkers et al. 2003), we find black-body radii of up to $280 \mathrm{~km}$, and we measure the rate at which the black-body radius increases to be $(1.6 \pm 0.6) \times 10^{4} \mathrm{~km} \mathrm{~s}^{-1}$, which are similar to the values found for two other superexpansion bursts (in't Zand \& Weinberg 2010). Including corrections can lead to $40 \%$ higher values (Section 3.1.4). The rate of black-body radius increase may trace the velocity of the expanding photosphere, but deviation of the spectrum from a black body and the changing optical depth have to be taken into account to determine the true photospheric velocity. Furthermore, during the strong radius expansion phase variations in the persistent flux may be present, which have not been accounted for.

\subsection{Variability in the superburst light curve}

Achromatic variability is present in the tail of the superburst of $4 \mathrm{U} 1820$-30 between 3365 - 7200 s after the onset (Strohmaver \& Brown 2002). This variability is suggested to result from alternate blocking and reflecting of burst emission by density inhomogeneities in the accretion disk, that are induced in the superexpansion phase (in 't Zand et al. 2011a). 
Shortly after the superexpansion period (around $47 \mathrm{~s}$ after onset or $33 \mathrm{~s}$ after the onset of superexpansion), another peak is present in the flux. The peak is also visible in the hardness ratio, so this is not achromatic variability and must be of a different origin. We also find the variability in the black-body parameters. These were, however, derived assuming a black-body spectrum, whereas the variability need not be of this nature. For example, the persistent background may vary, whereas we assume a constant background. in 't Zand et al. (2011a) estimate different timescales for processes in the disk. The timescale closest to that of the observed variability is the viscous timescale of approximately $60-950 \mathrm{~s}$ on which the accretion disk settles.

\subsection{Precursor from $4 U$ 1636-53}

The count rate for the superburst precursor of $4 \mathrm{U} 1636-53$ exhibits a dip similar to the light curve of the 4U 1820-30 superburst. Only a weak hint of PRE, however, may be visible in the hardness ratio. The quality of the data at the onset of the superburst is insufficient to argue for the presence of absence of radius expansion. The total photon counts are significantly lower for the precursor than for three short bursts in the preceeding month. Unlike the precursor of the $4 \mathrm{U}$ 1820-30 superburst, therefore, this precursor is not more energetic than what can be provided by nuclear burning in the atmosphere. Cumming et al. (2006) derive for this superburst an ignition depth that is just above the minimum required to generate a shock (Keek \& Heger 2011; see also Weinberg et al. 2006), and the models of Keek \& Heger (2011) that best fit the light curve do not produce a shock at all. The precursor would, therefore, mostly be powered by the burning of the available hydrogen and helium in the envelope, the amount of which depends on the time since the previous short burst, which is unknown. This may explain the lack of a powerful precursor and a strong PRE phase.

\subsection{Outlook}

The time resolution of $0.125 \mathrm{~s}$ was insufficient for fully resolving the start of the radius-expansion phase of the precursor from $4 \mathrm{U} 1820-30$, and the collecting area was too small to study the $4 \mathrm{U} 1636-53$ precursor. A future mission such as the Large Observatory for X-ray Timing
(LOFT, Feroci et al. 2011) is required to study precursors in detail. This will allow us to confirm the PRE nature in other superbursts, and provide important information on the detonation process deep in the neutron star envelope.

\section{CONCLUSIONS}

Using data from the propane detector of the PCA on $R X T E$, we perform the first detailed spectral analysis of superburst precursors. For the PCA superburst from $4 \mathrm{U} 1636-53$ the data are of insufficient quality, but for the PCA superburst from $4 \mathrm{U}$ 1820-30 the precursor clearly exhibits PRE. This confirms that the precursor is a single peak in the (bolometric) emission, and the dip in the peak is due to the limited band pass of the instrument.

The precursor from $4 \mathrm{U} 1820-30$ is $1.4-2$ times more energetic than the most powerful short X-ray burst we analyzed from the same source. This shows that the thermonuclear burning of helium in the atmosphere is insufficient to power the precursor. We suggest shock heating as the most likely additional contributor to the energetics. This is strong support for recent numerical models that predict superbursts to proceed as a detonation, and that the generated shock deposits enough heat in the atmosphere to power a bright precursor burst.

With the same technique we study the superexpansion phase of the $4 \mathrm{U} 1820-30$ superburst in greater detail then was previously possible. We find similar expansion factors as reported for the few other superexpansion bursts, and we derive expansion velocities of up to $5 \%$ of the speed of light.

The author thanks E. F. Brown and J. J. M. in 't Zand for comments on this paper, and E. Kuulkers for helpful discussions. This paper uses preliminary analysis results from the Multi-INstrument Burst ARchive (MINBAR), which is supported under the Australian Academy of Science's Scientific Visits to Europe program, and the Australian Research Council's Discovery Projects and Future Fellowship funding schemes. The author thanks the International Space Science Institute in Bern for hosting an International Team on Type I X-ray bursts. The author is supported by the Joint Institute for Nuclear Astrophysics (JINA; grant PHY08-22648), a National Science Foundation Physics Frontier Center.

\section{REFERENCES}

Asai, K., Dotani, T., Nagase, F., \& Mitsuda, K. 2000, ApJS, 131, 571

Ballantyne, D. R., \& Strohmayer, T. E. 2004, ApJ, 602, L105

Chou, Y., \& Grindlay, J. E. 2001, ApJ, 563, 934

Cornelisse, R., Heise, J., Kuulkers, E., Verbunt, F., \& in 't Zand, J. J. M. 2000, A\&A, 357, L21

Cornelisse, R., et al. 2003, A\&A, 405, 1033

Cumming, A. 2003, ApJ, 595, 1077

Cumming, A., \& Bildsten, L. 2001, ApJ, 559, L127

Cumming, A., Macbeth, J., in 't Zand, J. J. M., \& Page, D. 2006, ApJ, 646, 429

Feroci, M., et al. 2011, Experimental Astronomy, 100

Galloway, D. K., Muno, M. P., Hartman, J. M., Psaltis, D., \& Chakrabarty, D. 2008, ApJS, 179, 360

Hoffman, J. A., Lewin, W. H. G., \& Doty, J. 1977, ApJ, 217, L23

in 't Zand, J. J. M., Cornelisse, R., \& Cumming, A. 2004, A\&A, 426,257

in 't Zand, J. J. M., Galloway, D. K., \& Ballantyne, D. R. 2011a, A\&A, 525, A111 in 't Zand, J. J. M., Kuulkers, E., Verbunt, F., Heise, J., \& Cornelisse, R. 2003, A\&A, 411, L487

in 't Zand, J. J. M., Serino, M., Kawai, N., \& Heinke, C. 2011b,

The Astronomer's Telegram, 3625, 1

in't Zand, J. J. M., \& Weinberg, N. N. 2010, A\&A, 520, A81

Jahoda, K., Markwardt, C. B., Radeva, Y., Rots, A. H., Stark, M. J., Swank, J. H., Strohmayer, T. E., \& Zhang, W. 2006, ApJS, 163, 401

Keek, L., Galloway, D. K., in 't Zand, J. J. M., \& Heger, A. 2010, ApJ, 718, 292

Keek, L., \& Heger, A. 2011, ApJ, 743, 189

Keek, L., Heger, A., \& in 't Zand, J. J. M. 2012, ApJ, 752, 150

Keek, L., \& in 't Zand, J. J. M. 2008, in Proceedings of the 7th INTEGRAL Workshop. 8 - 11 September 2008 Copenhagen, Denmark. Online at

http://pos.sissa.it/cgi-bin/reader/conf.cgi?confid $=67$, p.32

Kuulkers, E. 2009, The Astronomer's Telegram, 2140, 1

Kuulkers, E., den Hartog, P. R., in 't Zand, J. J. M., Verbunt, F. W. M., Harris, W. E., \& Cocchi, M. 2003, A\&A, 399, 663 
Kuulkers, E., Homan, J., van der Klis, M., Lewin, W. H. G., \& Méndez, M. 2002a, A\&A, 382, 947

Kuulkers, E., in 't Zand, J., Homan, J., van Straaten, S., Altamirano, D., \& van der Klis, M. 2004, in AIP Conf. Proc. 714: X-ray Timing 2003: Rossi and Beyond, 257-260

Kuulkers, E., et al. 2002b, A\&A, 382, 503

Levine, A. M., Bradt, H., Cui, W., Jernigan, J. G., Morgan, E. H., Remillard, R., Shirey, R. E., \& Smith, D. A. 1996, ApJ, 469, L33+

Lewin, W. H. G., Vacca, W. D., \& Basinska, E. M. 1984, ApJ, $277, \mathrm{~L} 57$

Lewin, W. H. G., van Paradijs, J., \& Taam, R. E. 1993, Space Science Reviews, 62, 223

Morrison, R., \& McCammon, D. 1983, ApJ, 270, 119

Priedhorsky, W., \& Terrell, J. 1984, ApJ, 284, L17

Rappaport, S., Ma, C. P., Joss, P. C., \& Nelson, L. A. 1987, ApJ, 322,842
Revnivtsev, M., Churazov, E., Gilfanov, M., \& Sunyaev, R. 2001, A\&A, 372, 138

Stella, L., Priedhorsky, W., \& White, N. E. 1987, ApJ, 312, L17

Strohmayer, T. E., \& Brown, E. F. 2002, ApJ, 566, 1045

Strohmayer, T. E., \& Markwardt, C. B. 2002, ApJ, 577, 337

Suleimanov, V., Poutanen, J., \& Werner, K. 2011, A\&A, 527, A139+

Swank, J. H., Becker, R. H., Boldt, E. A., Holt, S. S., Pravdo, S. H., \& Serlemitsos, P. J. 1977, ApJ, 212, L73

Tawara, Y., et al. 1984, ApJ, 276, L41

van Paradijs, J., Sztajno, M., Lewin, W. H. G., Trumper, J., Vacca, W. D., \& van der Klis, M. 1986, MNRAS, 221, 617

Weinberg, N. N., \& Bildsten, L. 2007, ApJ, 670, 1291

Weinberg, N. N., Bildsten, L., \& Brown, E. F. 2006, ApJ, 650, L119

Wijnands, R. 2001, ApJ, 554, L59

Zdziarski, A. A., Gierliński, M., Wen, L., \& Kostrzewa, Z. 2007, MNRAS, 377, 1017 